$\xi=1$ 国

\title{
Improved hybrid inter and intra-cell interference cancellation mechanism for LTE-A HETNETS
}

\author{
Shibu $\mathrm{S}^{1}$, Saminadan $\mathrm{V}^{2}$ \\ ${ }^{1}$ Research ScholarDepartment of Electronics and Communication Engineering, Pondicherry Engineering College, \\ Puducherry 605014, India \\ ${ }^{2}$ ProfessorDepartment of Electronics and Communication Engineering,Pondicherry Engineering College, \\ Puducherry 605014, India \\ *Corresponding author E-mail: soman.shibu@gmail.com
}

\begin{abstract}
In Heterogeneous networks (HetNets), operators need to ensure reliable service to their users in terms of network performance and low cost. The growing demand of user traffic necessitates the incorporation of femto cells and pico cells for facilitating seamless services. The network performance of Long Term Evolution (LTE) and LTE Advanced (LTE-A) HetNets is drastically influenced by the degree of interference that is realized in the inter-cell and intra-cell during communication. In this paper, an Improved Hybrid inter and intra-cell interference cancellation mechanism (CRS-IIC-LFLS) is propounded for handling interference at the inter and intra-cell level. Inter-cell interference cancellation is achieved through the use of an Improved CRS-IIC algorithm that uses the benefits of Linear Filtering Least Square (LFLS) method for channel estimation. CRS-IIC-LFLS is found to potent in rapid interference cancellation facilitation by suppressing the noise level, reducing the impact of fading channel and inherently eliminates small energy aware paths from large energy aware paths. The Simulation results confirm that the proposed scheme ensures better network throughput under light load, medium load and heavy load conditions of the network. The results prove that the performance of CRS-IIC-LFLS with CRS-IC-LFLS and CRS-ICLLS based on spectral efficiency is improved at a mean rate of $21 \%$ and $26 \%$ respectively. The simulation results also portrays that the increase in the spectral efficiency of CRS-IIC-LFLS (64-QAM) is phenomenal to a maximum improvement of $12 \%$ over CRS-IIC-LFLS (16-QAM).
\end{abstract}

Keywords:LTE; Het Nets; CRS; LFLS.

\section{Introduction}

In the recent decade, it is realized that the data traffic over cellular networks has faced a dramatic and explosive growth. With the advent of novel and effective technologies, various schemes have been explored and investigated for improving the spectral efficiency in HetNets that consists of macro-cells and number of pico or femto cells [1]. From the literature, five significant issues has been identified for exploration in the special kind of HetNet called Ultra-Dense Network (UDN) in which majority of the pico-cell are positioned in very close proximity to the mobile stations for enhancing or upgrading capacity of the network through improved throughput [2-3].

The first issue focuses on the concept of offloading, the second issue details on the exploration of achieving enhanced spectrum efficiency, the third issue pertains to extension of spectrum, the fourth issue is related to the improvement in node density of the network and finally the fifth issue of interference is carefully explored. Generally, the frequency reuse parameter may be considered for enhancing the spectrum efficiency for facilitating the macro-cells and pico cells to share a common frequency [4-7]. But the off-load data rate and coverage range of the network may increase drastically as the macro-cell provides basic service with pico cell as the associating cell for co-ordination [8-10]. Thus the UEs associated by the pico cell are highly influenced by the RF signal referred as the inter-cell interference. This inter-cell inter- ference is mainly due to the high energized macro-cell located in neighborhood proximity of UEs associated through the pico cell. This problem of inter-cell interference is also maximized when the UEs lies within the cell coverage regions of macro-cells [11-13]. In the literature, significant numbers of schemes are propounded for handling intra-cell and inter-cell interference in HetNets. Among the potential contributions, enhanced inter-cell interference coordination (eICIC) is contributed for mitigating interference [14] thorough the biasing feature of signal strength that coordinates with the pico-cell for minimizing the power of interference to a potential limit. In eICIC, the macro-cell remains in sleep state until a time duration referred as ABS (Almost Blank Subframe) and thus interference is cancelled since the interacting UEs does not receive the signal from the shared channel of the physical downlink[15-18]. However, the cell specific reference signal (CRS) can be still received in eICIC.But the performance of the network gets degraded to a greater extent. Then, a number of interference cancellation schemes (CRS-IC) were proposed in the literature for estimating the channel of interference and then cancelling them through CRS-IC-LFLS and CRS-IC-LLS schemes [19-20].

The CRS-IC-LFLS and CRS-IC-LLS approaches used a strategy of log-likelihood ratio based muting and puncturing method. They utilized a receiver algorithm that has the potential of integrating interference cancellation scheme with direct decision oriented estimation of channel that resolves the issue of non-colliding CRS. Finally, CRS-IC-LLS is an efficient equalization method proposed 
for comparing its performance with the classical CRS IC methodology that possess lower complexity and more latency. Further, the timing and frequency offset decreases the network performance to a maximum degree under the impact of non-colliding CRS situation. But still, it lacks in achieving better throughput and spectral efficiency under possible network load conditions. Thus an Improved Hybrid inter and intra-cell interference cancellation mechanism is propounded for handling interference at the inter and intra-cell level. Inter-cell interference cancellation is achieved through the use of an Improved CRS-IIC algorithm that uses the benefits of Linear Filtering Least Square (LFLS) method for channel estimation

The remaining section of the paper is organized as follows: Section 2 represents the system model of HetNets used for investigating the performance of CRS-IIC-LFLS. Section 3 and 4 depicts the steps involved in the cancellation of inter and intra-cell interference of HetNets through LFLS and enhanced joint transmission scheme. The simulation setup and comparative results evaluation and investigation are portrayed with the cause and reasons of the superior performance of CRS-IIC-LFLS in section 5. Section 6 highlights the major contributions and merits of the CRS-IICLFLS over the benchmarked interference cancelation schemes.

\section{System model}

The system model used for implementing LFLS-based Improved CRS-IIC algorithm consists of single M-eNB with 'P' pico cells, ' $\mathrm{L}$ ' macro cell MUEs and ' $\mathrm{N}$ ' number of pico-user equipments. The system model is designed such that all the e-NBsare forced to use a single frequency spectrum in order to enhance the spectrum efficiency of the network. It is assumed that ' $N$ ' numbers of PUEs in the pico cell are distributed arbitrarily in a random manner. The main concentration of the proposed work focuses on the downlink transmission. In the proposed model, each M-eNB is connected to several ' $\mathrm{L}$ ' numbers of macro-cell MUEs, each P-eNB is connected to several ' $\mathrm{N}$ ' number of pico-cell PUEs and further, the concept of user association is neglected in the proposed work. The idea of user association is mainly neglected for the following reasons. Firstly, Maximum Reference Signal Reference Power (RSRP) plays a significant role for estimating user association in macro cell but the pico cells traditionally utilize the pico or femtoeNBs that facilitate smaller coverage area and enable low power for transmission. Secondly, the classical use of maximum RSRP induces unbalanced load among the cells of pico and macro under user association. Thirdly, when the UEs need to connect with P-eNBs, biasing based cell association technique must be ensured for resolving the load balancing problem and this method of cell association lead to reduced cell range expansion. Thus cell range expansion schemes have to be integrated with any interference cancellation scheme for improving the throughput and spectrum efficiency of the network. Most of the cell range expansion schemes of the literature handles load balancing but fails in ensuring optimal spectrum efficiency. Hence, a proposed scheme needs to be formulated in such a way to handle both inter-cell and intracellular interference cancellation. In this research, the influence in optimal expansion of cell range is also considered for analysis as it increases the spectrum efficiency to a considerable degree. The proposed CRS-IIC-LFLS is analyzed with both 64-QAM and 16QAM.

In the next section, the key techniques involved in inter and intracell interference cancellation through CRS-IIC-LFLS is detailed with their significances and limitations.

\section{LFLS Based improved CRS-IIC algorithm for inter-cell interference cancellation}

In the proposed system model, the virtual cell and the associated M-eNB are assumed to share a single frequency so as to enhance the frequency efficiency. Especially, this kind of interference is considered to be insignificant when the PUE receives the weak signal from the M-eNB and when they exist in the central proximity of the virtual cell. But, the degree of interference between the M-eNB and PUEs is highly impactful when the PUEs have a relative movement towards the edges of the virtual cell. This type of inter-cell interference management can be resolved using an Improved Interference Cancellation (IIC) algorithm that can effectively reduce the influence of interference to the potential level. This IIC algorithm is incorporated in the HetNets based on three novel steps. In the first step, the estimation and compensation of interference signals frequency and timing offset is determined. Secondly, the channel statistics is elucidated based on inference channel response estimated based on LFLS channel estimation approach. The LFLS scheme facilities the objective of suppressing noise to a significant threshold by choosing suitable linear filters that can improve the system performance under multi-path channel constraints.

\subsection{Frequency and timing offset based channel estima- tion and compensation}

In this first phase of IIC, the compensation and estimation of channel in the time domain is achieved through the concept of cross-correlation of PSS/SSS symbols using (1) as

$$
\{\Delta \hat{m}, \Delta \hat{f}\}=\underset{\Delta m, \Delta f}{\arg \max } x(K|C(\Delta m, \Delta f)|)
$$

Where

$$
K(\Delta m \cdot \Delta f)=\sum_{n=1}^{M} S_{i}(n) r(\Delta m+n) e^{(-2 \pi \Delta f m / M)}
$$

The kind of cross-correlation are mainly used as it possess the potential of synchronization through the second last and last symbols present in the slot 0 and slot 10 of PSS/SSS symbols. Even though most of the algorithms proposed for channel estimation and compensation relies on synchronizing frequency and timing offset, this IIC uses the time domain signal nature using the basis of Cyclic Prefix (CP) [16]. The data resource elements of the macro-cell seems to be zero in ABS as they reduce the energy utilization rate of $\mathrm{CP}$ to a drastic level and hence the phenomenal decrease in $\mathrm{CP}$ hurdles the synchronization of frequency and timing to an influential level

\subsection{Estimation of interfering channel response using LFLS}

In IIC, the reconstruction of interfering signal is achieved only based on the estimation of interfering channel response. In order to simplify the computation complexity and improve accuracy, LFLS channel estimation technique [17] is used. In LFLS, initially Channel Frequency Response (CFR) is estimated based on block type pilots. Then linear filtering is incorporated for channel estimation. The LFLS channel estimation technique filters the AWGN by facilitation a wavelet kind of filtering in the timing domain. The channel estimation estimated through LFLS channel estimation technique is represented through (3) as

$$
\hat{g}_{L F L S}(m)=\frac{\hat{g}_{L L S}(m) *\left|\hat{g}_{L L S(m)}\right|^{2}}{\eta *\left|\hat{g}_{L L S}(m)\right|^{2}+(1-\eta) C^{2}}
$$

Where ' $\mathrm{C}$ ' and ' $\eta$ 'represent the channel impulse response ( $\left.C=\max _{0 \leq m \leq L}\left\{\left|\hat{g}_{L L S}(m)\right|\right\}\right)$ and suppression parameter of the incorporated linear filter. The utilized LFLS channel estimation technique is preferred over LLS channel estimation technique [18] as it is superior in synchronization and improving the estimation of interfering channel response. In IIC scheme, the replacement of the interfering CRS is made over the interference signal as the 
proposed scheme only relies on the interfering CRS signal of the macro cell into consideration. Further the mean value of the interfering channel can be computed through the use of time domainbased moving average window method. Hence the approximation of ' $\hat{g}_{L F L S}(m)$ 'is achieved by computation the expectation of $E\left(\hat{g}_{L F L S}(m)\right)$ only when the length of the moving average window is less than the channel coherence time.

In IIC, LFLS is used for the following benefits,

a) It not only suppresses the noise level but also suppresses the small energy aware paths to a significant degree.

b) It reduces the influence of fading channel and thereby enhances the channel estimation accuracy to a phenomenal extent.

c) It reduces the demerit of LLS channel estimation as they possess an inherent capability of differentiating small energy aware paths from large energy aware paths.

\subsection{Reconstruction of interfering channel response us- ing local CRS}

Similar to IC, IIC reconstructs the interference signal through the utilization of local CRS in the time domain when interfering channel estimation after TO/CFO is performed. In IIC, the reconstruction of interference signal is determined in the time domain and then the reconstructed signal is subtracted from the received signal through (4) as

$$
\hat{y}^{a}(m)=y(m)-\hat{x}(m+\Delta m) e^{(j 2 \pi \Delta \hat{f} m / N)} \otimes \hat{g}_{l}
$$

This reconstruction depends on ' $\hat{x}(\boldsymbol{m})$ ' referred as reconstructed interference CRS signal with' $\otimes$ ' as the circular convolution representation under the constraint specified in (5) as

$$
\hat{g}_{l}=\operatorname{IFFT}\left[\hat{H}_{i, k}^{(n)}\right]
$$

Hence, the interfering signal is reconstructed and the issue of inter-cell management is effectively and efficiently resolved.

\section{Virtual cell inhibited joint transmission technique for intra-cell interference man- agement}

Similar to inter-cell interference management, intra-cell interference management is handled through the utilization of virtual cell inhibited joint transmission technique proposed in [13]. Intra-cell interference is mainly due to the recent development of ultradense HetNet that diminishes the distance between pico cells. The proposed CRS-IIC-LFLS scheme experiences a special situation once the inter-cell cancellation mechanism has been incorporated as the strength of the signal is potentially enhanced under the increasing interference impact between pico cells degrades to a specific level. Hence, both the inter-cell and intra-cell interference cancellation schemes are necessary for improving the network performance. In this CRS-IIC-LFLS scheme, intra-cell interference between virtual cells are handled based on the concept of joint transmission, This joint transmission approach of intra-cell interference management defines some characteristic features of virtual cells that include the use of similar kind of states and reduced distance between pico cells. This concept of virtual cell based joint transmission is similar to the joint transmission scheme of the CoMP as the they choose a potential PUEs as the coordinating user when that specific PUEs receive much stronger signals from a large number of P-eNBs and they can be utilized for connecting a number of coordinating eNBs at the same time, the PicoeNBs that facilitate a much stronger signal is selected as the coordinating eNBs. In this intra-cell management scheme, the details of the physical layer are ignored as this interference cancellation technique focuses only on the impacts of joint transmission like multiple beamforming. In the real time situation, the pico-eNBs that interfere with the other e-NBs can be selected as the coordinating eNBs. Once the co-ordinated UEs are selected based on the PUE that is greatly impacted under the influence of neighboring Pico-eNBs are selected as the new co-ordinated e-NBs. When the PUEs are selected as coordinators, each one of them update RSRP list and the e-NBs that possess the optimal feasible RSRP is selected as the co-ordinate eNB to all the nodes neglecting the classical base station. The condition that needs to be satisfied for a PUE to be selected as the coordinator depends on equation (6) as mentioned below

$$
R S R P_{C R S-I C-L F L S}>R S R P_{\text {THRESHOLD }}(6)
$$

In this joint transmission scheme, the stronger signal received from the neighboring e-NB is used as the signal of transmission rather than the interfering signal. Hence the receiver power of the coordinating PUEs is considered to be equal to the initial interference power actually influenced. In contrast, if the strength of the signal received from the neighboring e-NBs are weak and the influence of interference induced by them is not severe then the corresponding PUE remains in connection with the current interacting P-eNBs. Thus the intra-cell interference is cancelled by CRS-IIC-LFLS to a significant threshold.

\section{Performance evaluation and analysis}

In this section, the performance of CRS-IIC-LFLS (64-QAM) is analyzed with CRS-IIC-LFLS (16-QAM), CRS-IC-LFLS and CRS-IC-LLS based on three experiments viz., i) Experiment-1Performance of CRS-IIC-LFLS (64-QAM) based on network load conditions by varying the number of users, ii) Experiment-2Performance of CRS-IIC-LFLS (64-QAM) based on Frequency offset and Time offset under varying number of users and iii) Experiment-3-Performance of CRS-IIC-LFLS (64-QAM) based on BER and BLER through SNR variation. This performance investigation is achieved based on performance metrics that include BER(Bit Error Rate), BLER (Block Error Rate), Throughput and Spectral efficiency under varying network load conditions and SNR(Signal to Noise Ratio).

\subsection{Simulation settings}

In this simulation experiment, 3GPP standardization system oriented simulation platform is built for investigating the performance of the proposed CRS-IIC-LFLS scheme with 64-QAM and 16-QAM and it also aids in comparing its performance with the existing CRS-IC-LFLS and CRS-IC-LLS schemes. To perform higher efficient simulation experiments, the distinct proportional fair scheduling scheme is incorporated for sustaining fairness as the significant numbers of users are randomly distributed in the cell coverage region and some of the peculiar process of protocol stack is modified suitably to focus the proposed algorithm towards possible kinds of network load. The proposed CRS-IIC-LFLS-64QAM, CRS-IIC-LFLS -16-QAM and CRS-IC-LFLS and CRS-ICLLS schemes are investigated under the same and peculiar deployment environment. In this proposed environment, one $\mathrm{M}$ ENBs and eight P-ENBs in one section, ' $L$ ' MUEs and ' $N$ ' PUEs located at the cell center and cell edge respectively. As mentioned in the system model, both the M-ENB and P-ENBs are made to share the same frequency in order to enhance the spectral efficiency of the proposed scheme.

Table 1: Simulation Parameters

\begin{tabular}{ll}
\hline Description & Parameters \\
\hline Bandwidth of M-eNB & $15 \mathrm{MHz}$ \\
Bandwidth of P-eNB & $15 \mathrm{MHz}$ \\
Cell radius of M-eNB & $600 \mathrm{~m}$ \\
Cell radius of P-eNB & $60 \mathrm{~m}$ \\
Traffic model used & Full-buffer \\
\hline
\end{tabular}




\begin{tabular}{ll}
\hline Bias for cell association & $10 \mathrm{~dB}$ \\
Transmit power for M-eNB & $48 \mathrm{~dB}$ \\
Transmit power for P-eNB & $32 \mathrm{~dB}$ \\
Duration for sub-frame & $1 \mathrm{msec}$ \\
Noise Figure & $145.98 \mathrm{~dB}$ \\
\hline
\end{tabular}

\subsection{Simulation results and analysis}

a) Experiment-1-Performance analysis of CRS-IIC-LFLS (64QAM) based on network load conditions by varying the number of users

In this experiment, the performance of CRS-IIC-LFLS (64-QAM), CRS-IIC-LFLS (16 QAM), CRS-IC-LFLS and CRS-IC-LLS are analyzed under different network load conditions that are classified into light load, medium load and heavy load. Figure 1, 2 and 3 depicts the performance of CRS-IIC-LFLS (64-QAM) over CRS-IIC-LFLS (16 QAM), CRS-IC-LFLS and CRS-IC-LLS based on throughput by varying the number of users under aforementioned network load conditions.

Fig.1. infers that the performance of CRS-IIC-LFLS (64-QAM) under light load is superior to CRS-IIC-LFLS (16 QAM) and the CRS-IC-LFLS and CRS-IC-LLS approaches as the interference cancellation technique facilitated by them are rapid and possess the ability of reducing the fading impacts. It is inferred that the throughput of CRS-IIC-LFLS (64-QAM) is improved by 7\%, 20\% and $32 \%$ when compared to the proposed CRS-IIC-LFLS (16 QAM) and the existing CRS-IC-LFLS and the existing CRS-ICLLS schemes available for interference cancellation. It is also proved that this phenomenal increase in throughput is mainly due to the incorporation of LFLS that potentially reduces the noise level to a maximum extent.

Further, Fig. 2. depicts that the performance of CRS-IIC-LFLS (64-QAM) compared CRS-IIC-LFLS (16 QAM), CRS-IC-LFLS and CRS-IC-LLS approaches under medium load. It is identified that the throughput of CRS-IIC-LFLS (64-QAM) is increased by $21 \%, 29 \%$ and $36 \%$ than the investigated CRS-IIC-LFLS (16 QAM) and the benchmarked CRS-IC-LFLS and CRS-IC-LLS techniques. This significant performance is mainly due to the utilization of LFLS and virtual cell based joint management scheme inspired based on RSRP. It is also found that the proposed scheme is potential enough in optimally selecting the coordinating e-NBs at a faster rate that induces rapid cancellation of interference both at the inter and intra-cell level.

Likewise, Fig. 3. presents the performance of CRS-IIC-LFLS (64QAM) under heavy load network conditions and the proposed scheme is found to be predominant to CRS-IIC-LFLS (16 QAM) and the CRS-IC-LFLS and CRS-IC-LLS approaches as they are capable of enforcing joint transmission technique at the intra-cell level and LFLS at the inter-cell level. It is identified that the performance of the network in terms of throughput is enhanced by CRS-IIC-LFLS (64-QAM) over CRS-IIC-LFLS (16 QAM) and the existing CRS-IC-LFLS and CRS-IC-LLS schemes by a phenomenal improvement of $17 \%, 24 \%$ and $28 \%$ respectively.

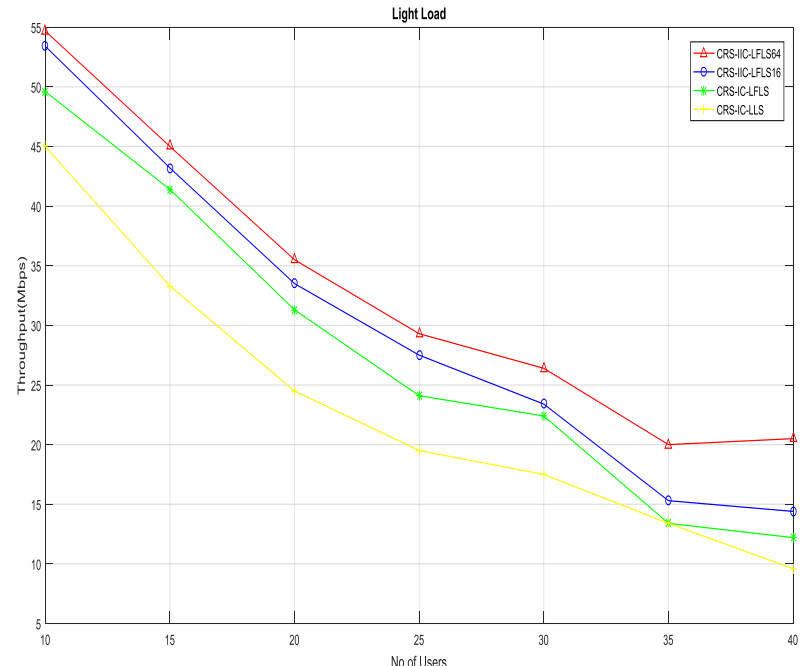

Fig. 1:Comparison for Throughput of CRS-IIC-LFLS (64-QAM) Under Light Load.

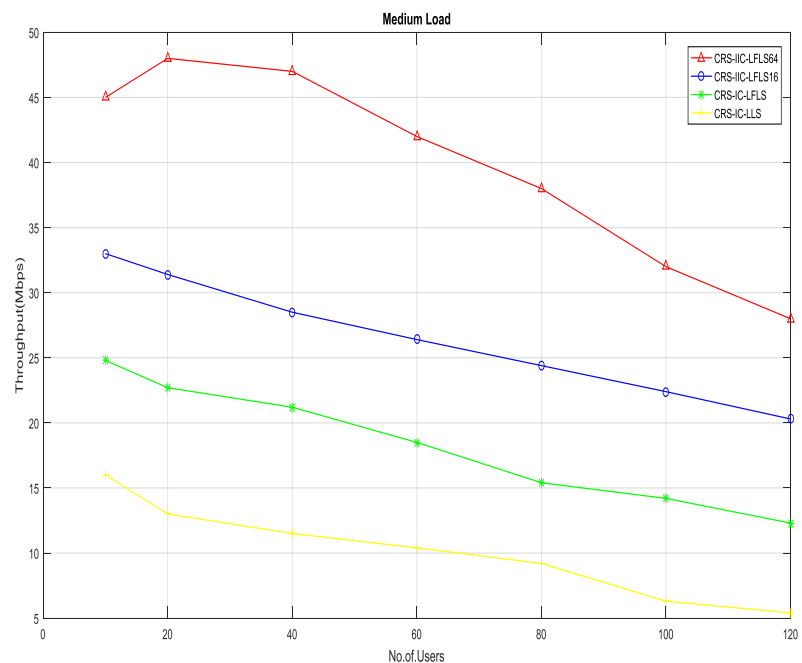

Fig. 2:Comparison for Throughput of CRS-IIC-LFLS (64-QAM) Under Medium Load.

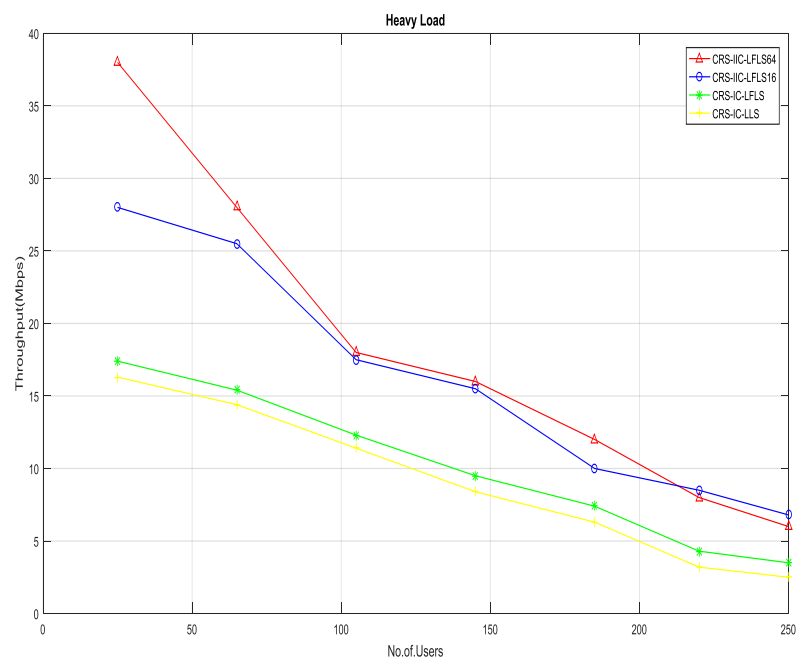

Fig. 3:Comparison for Throughput of CRS-IIC-LFLS (64-QAM) Under Heavy Load. 


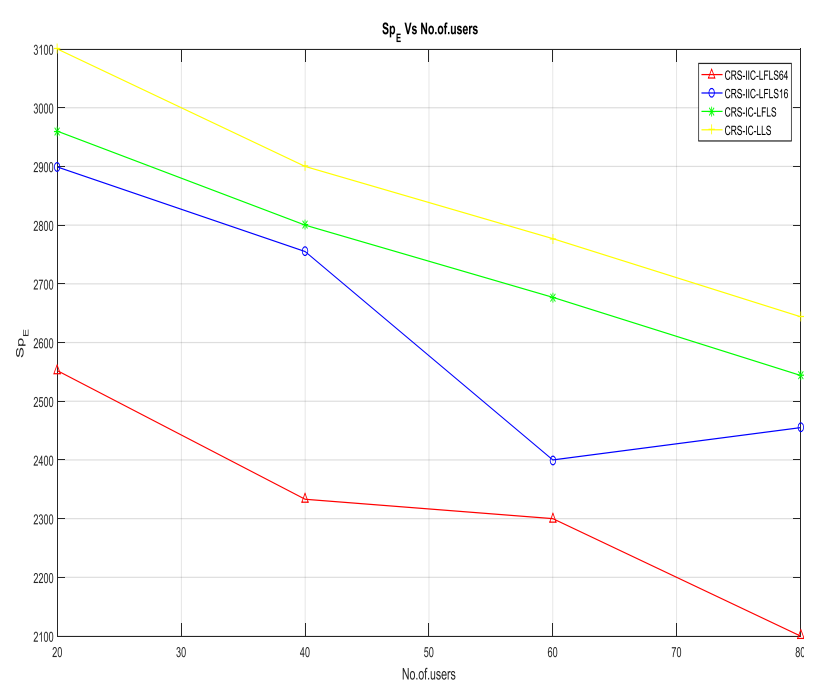

Fig. 4:Spectral Efficiency of CRS-IIC-LFLS (64-QAM) with Varying Number of Users.

In addition, Fig. 4 presents the improvement in spectral efficiency achieved by the proposed CRS-IIC-LFLS (64-QAM) over CRSIIC-LFLS (16 QAM), CRS-IC-LFLS and CRS-IC-LLS approaches. The spectrum efficiency of the proposed CRS-IIC-LFLS (64QAM) is effectively to a maximum level of $8 \%$ over CRS-IICLFLS (16 QAM), $28 \%$ over CRS-IC-LFLS and 33\% over CRSIC-LLS algorithms considered for investigation. This quantifiable degree of improvement in spectral efficiency of CRS-IIC-LFLS (64-QAM) and CRS-IIC-LFLS (16 QAM) over the existing schemes is mainly due to the deployment of virtual cell load policing approach that sustains the performance in par with the dynamic changing network load conditions.

b) Experiment 2- Performance analysis of CRS-IIC-LFLS (64QAM) based on Frequency offset and Time offset under varying number of users

In this experiment 2, the performance of CRS-IIC-LFLS (64QAM) and CRS-IIC-LFLS (16 QAM) are analyzed based on frequency offset and time offset. Fig .5 and 6 highlights the performance of CRS-IIC-LFLS (64-QAM) and CRS-IIC-LFLS (16 QAM) based on different CFO (Carrier Frequency Offset) and TO (Time Offset). From Fig. 5, it is obvious that the performance of CRS-IIC-LFLS (16 QAM) is superior to CRS-IIC-LFLS (64QAM) in terms of BLER. The improvement of CRS-IIC-LFLS (16 QAM) over CRS-IIC-LFLS (64 QAM) is about 22\%. It is also identified that both the CRS-IIC-LFLS (16 QAM) and CRS-IICLFLS (64 QAM) schemes are dominant in reducing the BLER rate to a maximum threshold of $27 \%$.

However, From Fig. 6, the performance of CRS-IIC-LFLS (64 QAM) is improved over CRS-IIC-LFLS (16 QAM) when the timing offset is varied at regular intervals. The improvement of CRS-IIC-LFLS (64 QAM) over CRS-IIC-LFLS (16 QAM) in terms of BLER with varying time offset is about $11 \%$. It is also confirmed the efficiency of CRS-IIC-LFLS (64 QAM) and CRSIIC-LFLS (16 QAM) schemes are dominant in reducing the BLER rate to a maximum threshold of $14 \%$.

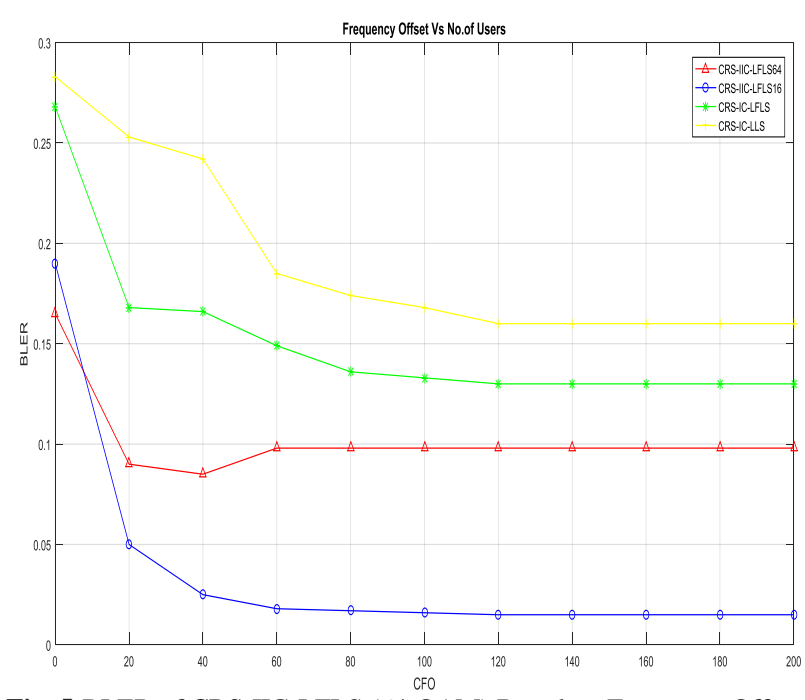

Fig. 5:BLER of CRS-IIC-LFLS (64-QAM) Based on Frequency Offset

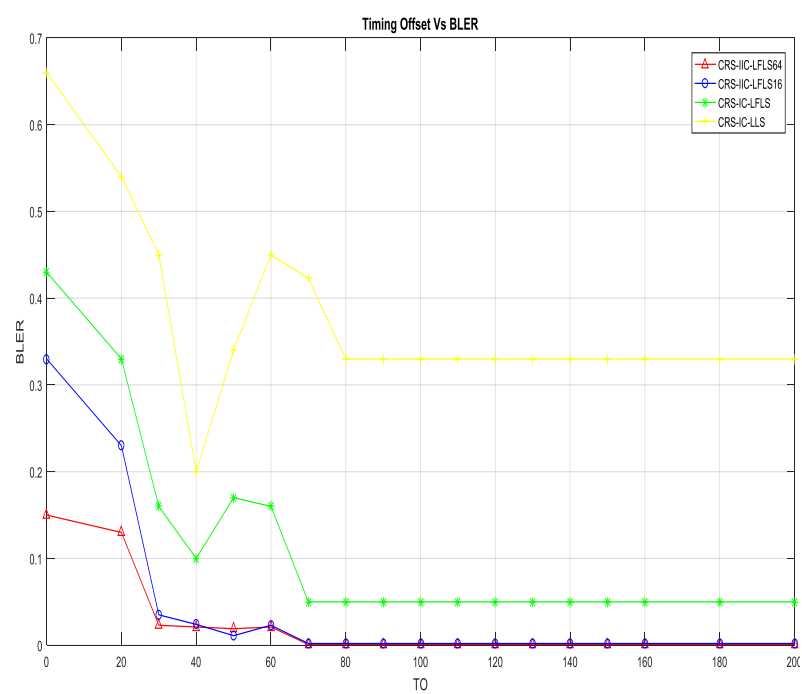

Fig. 6:BLER of CRS-IIC-LFLS (64-QAM) Based on Timing Offset

c) Experiment 3- Performance analysis of CRS-IIC-LFLS (64QAM) based on BER and BLER through SNR variation.

In this experiment, first the performance ofCRS-IIC-LFLS is analyzed based on BER and BLER with respect to change in SNRs and then the increase in percentage of spectrum efficiency achieved by the CRS-IIC-LFLS (64-QAM), CRS-IIC-LFLS (16 QAM), CRS-IC-LFLS and CRS-IC-LLS approaches are compared by varying the network load conditions. Fig. 7 and 8 highlights the phenomenal decrease of BER and BLER induced by CRS-IICLFLS (64 QAM) and CRS-IIC-LFLS (16 QAM) approaches over the existing CRS-IC-LFLS and CRS-IC-LLS schemes. The decrease in BER and BLER achieved by CRS-IIC-LFLS (64-QAM), CRS-IIC-LFLS (16 QAM) ranges to about $16 \%$ to $19 \%$ over the benchmark schemes used for comparative investigation. 


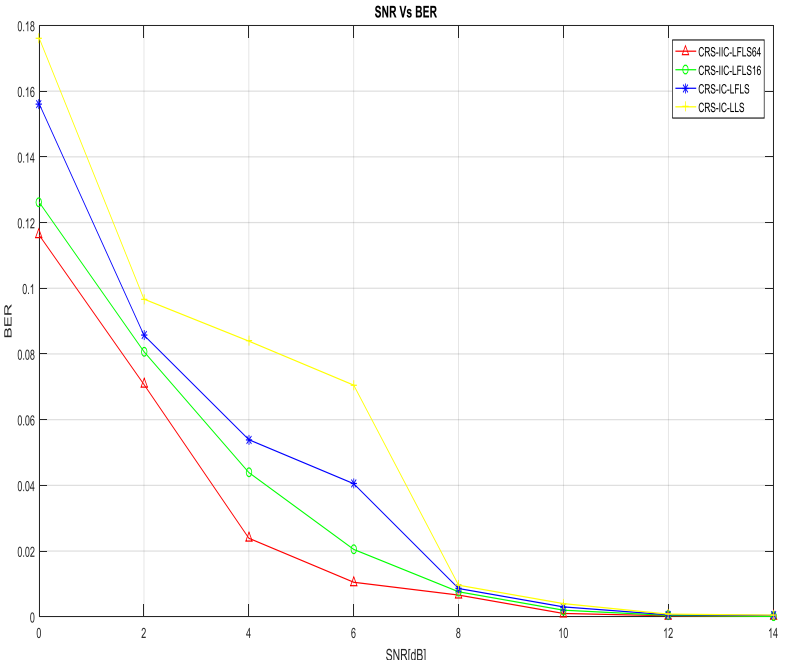

Fig. 7:BER of CRS-IIC-LFLS (64-QAM) Based on Varying SNR.

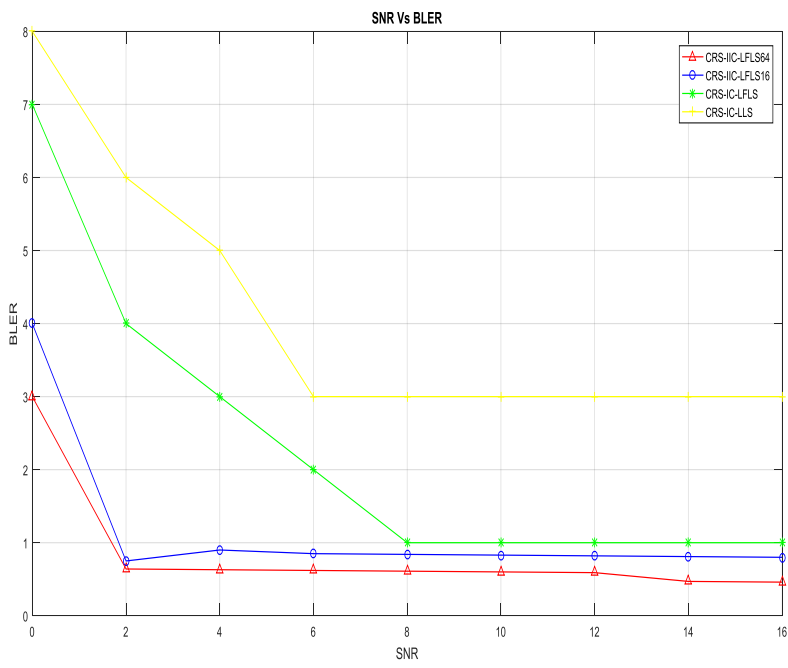

Fig. 8:BLER of CRS-IIC-LFLS (64-QAM) Based on Varying SNR.

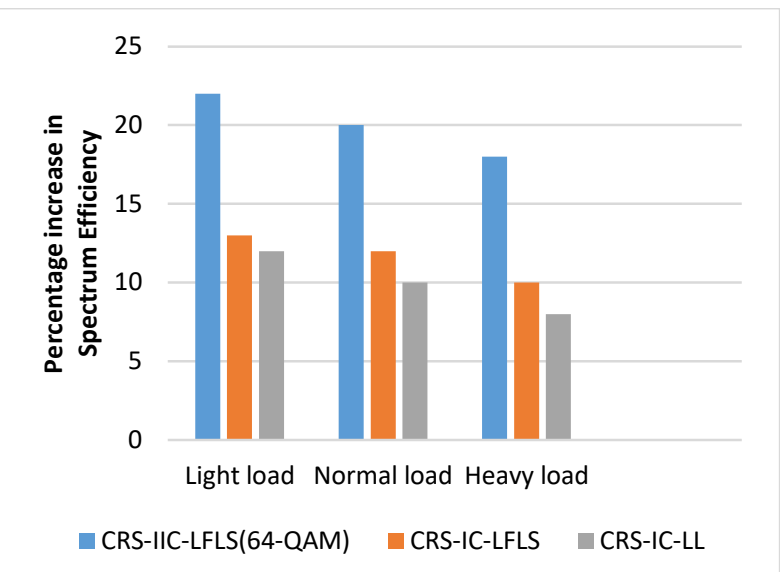

Fig. 9:Percentage Improvement in Spectral Efficiency of CRS-IIC-LFLS (64-QAM)

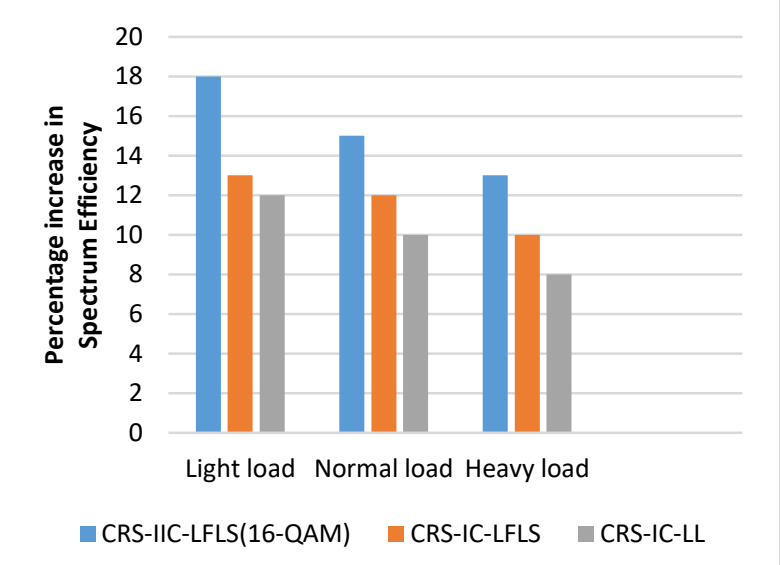

Fig. 10:Percentage Improvement in Spectral Efficiency of CRS-IIC-LFLS (16-QAM)

Fig. 9 and 10 portrays the percentage improvement of spectrum efficiency enabled by CRS-IIC-LFLS (64 QAM) and CRS-IICLFLS (16 QAM) approaches over the existing CRS-IC-LFLS and CRS-IC-LLS schemes. The percentage improvement in spectrum efficiency of CRS-IIC-LFLS (64 QAM) is about 19\% and 22\% superior to CRS-IC-LFLS and CRS-IC-LLS schemes. Likewise, The percentage improvement in spectrum efficiency of CRS-IICLFLS (16 QAM) is about $13 \%$ and $16 \%$ superior to CRS-ICLFLS and CRS-IC-LLS schemes. Finally, it is also proved that the increase in the spectral efficiency of CRS-IIC-LFLS (64-QAM) is phenomenal to a maximum improvement of $12 \%$ over CRS-IICLFLS (16-QAM).

\section{Conclusion}

In this paper, An Improved Hybrid inter and intra-cell interference cancellation mechanism called CRS-IIC-LFLS is formulated and investigated under 64-QAM and 16-QAM for suitable cancellation of interference impact in LTE-A HetNets. The proposed CRS-IICLFLS technique is identified to be predominant than the considered interference cancellation schemes for two main reasons. First, CRS-IIC-LFLS utilizes the LFLS channel estimation scheme for estimating interfering channel response and reconstructing the interfering signal through the local CRS utilization and second, they incorporate a joint transmission scheme that uses the benefits of virtual cell for selecting coordinated PUEs when their RSRP vale is below the predefined threshold for improving the throughput of the network. The simulation results prove that CRS-IICLFLS schemes incorporating 64 QAM and 16 QAM is found to sustain the throughput of the network to a maximum degree of $16 \%$ and $19 \%$ than the considered inference cancellation techniques used for comparison. The results also confirms that the spectral efficiency of CRS-IIC-LFLS (64-QAM) is phenomenal increased at a maximum level of $12 \%$ over CRS-IIC-LFLS (16QAM).

\section{References}

[1] Ye, Q., Rong, B., Chen, Y., Al-Shalash, M., Caramanis, C., \& Andrews, J. G. (2013). User Association for Load Balancing in Heterogeneous Cellular Networks. IEEE Transactions on Wireless Communications, 12(6), 2716. https://doi.org/10.1109/TWC.2013.040413.120676

[2] Rihan, M., Elsabrouty, M., Muta, O., \& Furukawa, H. (2015). Interference Alignment with Limited Feedback for Macrocell-Femtocell Heterogeneous Networks. 2015 IEEE 81st Vehicular Technology Conference (VTC Spring), 1(1), 23. https://doi.org/10.1109/VTCSpring.2015.7145883.

[3] Hunukumbure, M., \&Vadgama, S. (2015). A Hybrid Interference Control Scheme for Femto Cells Using Overhearing and Limited SON Co-Ordination. 2015 IEEE 81st Vehicular Technology Conference (VTC Spring), 2(1), 35-45. 
[4] Fu, S., Wu, B., Wen, H., Ho, P., \& Feng, G. (2014). Transmission Scheduling and Game Theoretical Power Allocation for Interference Coordination in CoMP. IEEE Transactions on Wireless Communications, 13(1), 123. https://doi.org/10.1109/TWC.2013.120113.121646.

[5] Qiang, L., Yang, Y., Shu, F., \& Gang, W. (2010). Coordinated beamforming in downlink CoMP transmission system. Proceedings of the fifth International ICST Conference on Communications and Networking in China, 1(1), 23 34. https://doi.org/10.4108/chinacom.2010.151.

[6] Sun, Y., Wang, Z., Wang, T., Wu, Y., \& Fang, Y. (2016). Joint Optimization of Parameters for Enhanced Inter-Cell Interference Coordination (eICIC) in LTE-A HetNets. IEICE Transactions on Communications, 3(2), 23-41.

[7] Mogensen, P., Na, W., Kovacs, I. Z., Frederiksen, F., Pokhariyal, A., Pedersen, K. I, Kuusela, M. (2007). LTE Capacity Compared to the Shannon Bound. 2007 IEEE 65th Vehicular Technology Conference - VTC2007-Spring, 2(1), 23-30.

[8] Visotsky, E., Mondal, B., Thomas, T. A., Mangalvedhe, N., Ghosh, A., \& Wang, X. (2013). Joint Scheduling for CoMP and eICIC in Heterogeneous Network Deployments. 2013 IEEE 77th Vehicular Technology Conference (VTC Spring), 2(1), 3548. https://doi.org/10.1109/VTCSpring.2013.6692613.

[9] Liu, A., Lau, V. K., Ruan, L., Chen, J., \& Xiao, D. (2014). Hierarchical Radio Resource Optimization for Heterogeneous Networks with Enhanced Inter-Cell Interference Coordination (eICIC). IEEE Transactions on Signal Processing, 62(7), 16841693. https://doi.org/10.1109/TSP.2014.2302748.

[10] Vasudevan, S., Pupala, R. N., \&Sivanesan, K. (2013). Dynamic eICIC - A Proactive Strategy for Improving Spectral Efficiencies of Heterogeneous LTE Cellular Networks by Leveraging User Mobility and Traffic Dynamics. IEEE Transactions on Wireless Communications, 12(10), 4956 4969.https://doi.org/10.1109/TWC.2013.081413.121651.

[11] Zhou, X., Yang, F., \& Song, J. (2012). Novel Transmit Diversity Scheme for TDS-OFDM System With Frequency-Shift mSequence Padding. IEEE Transactions on Broadcasting, 58(2), 317-324. https://doi.org/10.1109/TBC.2012.2186729.

[12] Feng, C., Cui, H., Ma, M., \& Jiao, B. (2013). On Statistical Properties of Co-channel Interference in OFDM Systems. IEEE Commu2331. https://doi.org/10.1109/LCOMM.2013.101813.131297.

[13] Ming Huang \& Wen Xu. (2012). Macro-femto inter-cell interference mitigation for 3GPP LTE-A downlink. 2012 IEEE Wireless Communications and Networking Conference Workshops (WCNCW), 3(1) 65.https://doi.org/10.1109/WCNCW.2012.6215544.

[14] Deb, S., Monogioudis, P., Miernik, J., \& Seymour, J. P. (2014). Algorithms for Enhanced Inter-Cell Interference Coordination (eICIC) in LTE HetNets. IEEE/ACM Transactions on Networking, 22(1) 137-150. https://doi.org/10.1109/TNET.2013.2246820.

[15] Zhang, H., Chen, S., Li, X., Ji, H., \& Du, X. (2015). Interference management for heterogeneous networks with spectral efficiency improvement. IEEE Wireless Communications, 22(2), 101107. https://doi.org/10.1109/MWC.2015.7096292.

[16] Montojo, J. I., \& Milstein, L. B. (2011). Error Rate for PSK and QAM Modulations for Non-Ideal OFDM Systems with Noisy Channel Estimates and Receive Diversity. IEEE Transactions on Communications, 59(10), 27032715. https://doi.org/10.1109/TCOMM.2011.081210.090609.

[17] Krishna, E. H., Sivani, K., \& Reddy, K. A. (2014). On the use of singular spectrum analysis for OFDM, channel estimation. 2014 International Conference on Electronics and Communication Systems (ICECS), 2(1), 15-25. https://doi.org/10.1109/ECS.2014.6892792.

[18] Yang Nan, Xin Sun, \& Li Zhang. (2015). Near-optimal pilot allocation in sparse channel estimation for massive MIMO OFDM systems. 2015 International Symposium on Wireless Communication Systems (ISWCS), 3(3), 45-62.

[19] Yang Zhang, Xin Zhang, \&Dacheng Yang. (2013). A robust least square channel estimation algorithm for OFDM systems under interferences. 2013 IEEE Wireless Communications and Networking Conference (WCNC), 2(1), 3448.https://doi.org/10.1109/WCNC.2013.6555061.

[20] You-Seok Lee, Hyun-Chool Shin, \&Hyoung-Nam Kim. (2009). Channel Estimation Based on a Time-Domain Threshold for OFDM Systems. IEEE Transactions on Broadcasting, 55(3), 656662. https://doi.org/10.1109/TBC.2009.2027575. 